\title{
INJECTION AND EXTRACTION LINES FOR THE ILC DAMPING RINGS*
}

\author{
I. Reichel ${ }^{\dagger}$, LBNL, Berkeley, CA 94720, USA
}

\section{Abstract}

The current design for the injection and extraction lines into and out of the ILC Damping Rings is presented as well as the design for the abort line. Due to changes of the geometric boundary conditions by other subsystems of the ILC, a modular approach has been used to be able to respond to recurring layout changes while reusing previously designed parts.

\section{INTRODUCTION}

\section{ILC layout}

In the ILC, electrons and positrons are produced and accelerated to an energy of $5 \mathrm{GeV}$ before being injected into the damping rings. The damping rings are required to decrease the emittance of the beam in order to achieve the desired luminosity. After extraction from the damping rings, the beams are transported through the RTML into the main linac.

The original design for the ILC had the damping rings housed in tunnels on either end of the main linac (see Fig. 1). During the time the injection and extraction lines were first designed, the details of the layout, i.e., the position of the damping ring with respect to the source and the RTML (which connects it to the main linac) kept changing. This required frequent changes of the layout of the injection and extraction lines.

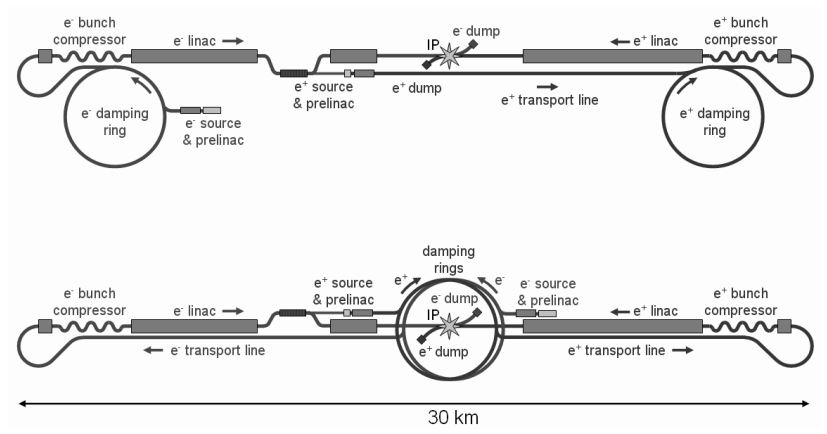

Figure 1: ILC layout with damping ring tunnels at both ends of the main linac (top) and one central damping ring tunnel (bottom).

For cost reasons it was later decided to house both damping rings in a common tunnel at the center of the facility

\footnotetext{
* This work was supported by the U.S. Department of Energy under Contract No. DE-AC02-05CH11231.

$\dagger$ ireichel@lbl.gov
}

(see Fig. 1), necessitating a completely different layout of the lines.

\section{Injection Line}

The injection line takes the beam from the bunch compressor into the damping ring. The part in the ring consists of two septa and 42 kicker magnets. The kickers are placed at four different locations with quadrupole magnets in between them. Their location within the long straight section of the damping rings changed several times.

This system is usually studied in reverse, as the part in the ring is given by constraints from the ring. It is evaluated as if it were an extraction line. At the end, the lattice is simply reversed.

\section{Extraction Line}

The extraction line takes the beam from the damping ring to the RTML. The part in the ring consists of 22 kickers and two septa. For the original layout with separate tunnels for the two damping rings, the extraction was located downstream from the injection in the same straight section. In the layout with a single damping ring tunnel, the extraction is located in the opposite straight section from the injection.

\section{Abort Line}

The aim of the abort line is to extract the entire beam cleanly from the ring and to expand the beam size enough to not destroy the dump. The nominal emittances of the fully damped beam are $\varepsilon_{x}=500 \mathrm{pm}$ and $\varepsilon_{y}=2 \mathrm{pm}$. The energy spread is $\frac{\Delta E}{E}=1.3 \times 10^{-3}$. The beam size on the dump must be of the order of $1 \mathrm{~mm}$ horizontally and vertically in order to avoid damage to the dump.

Kickers and septa are assumed to be similar to the ones used for injection and extraction for this study.

Increasing the beam size by mismatching the line requires a significant number of quadrupole magnets and the abort line would be $\mathcal{O}(100 \mathrm{~m})$ long after the septum, as the vertical $\beta$-function needs to be about $500 \mathrm{~km}$. As the horizontal beam size expands quickly due to the large dispersion from kickers and septa, the simplest approach to the abort line is to also introduce vertical dispersion. This is done using one vertical bending magnet after the septum. The magnet used is a type used in the RTML. This is, by design, a horizontal bending magnet rotated by $90^{\circ}$ with respect to its standard orientation.

Layouts for two different vertical bending angles have been studied, $150 \mathrm{mrad}$ and $75 \mathrm{mrad}$. Table 1 shows the 
Table 1: Approximate positions and angles of end of abort line for two different vertical bending angles with respect to end of septum. Note that the coordinate system is different from the one used for the other lines (origin at the end of the septum instead of in the center of the straight).

\begin{tabular}{crrrrr} 
bending angle & length & $x^{a}$ & $x^{\prime}$ & $y^{b}$ & $y^{\prime}$ \\
\hline $75 \mathrm{mrad}$ & $12.4 \mathrm{~m}$ & $1.45 \mathrm{~m}$ & $110.8 \mathrm{mrad}$ & $0.75 \mathrm{~m}$ & $75 \mathrm{mrad}$ \\
$150 \mathrm{mrad}$ & $9.4 \mathrm{~m}$ & $1.05 \mathrm{~m}$ & $110.8 \mathrm{mrad}$ & $1.05 \mathrm{~m}$ & $150 \mathrm{mrad}$ \\
\hline \hline
\end{tabular}

${ }^{a}$ Horizontal displacement of beam at the end of the line.

${ }^{b}$ Vertical displacement of beam at the end of the line.

coordinates of the end of the line with respect to the end of the septum in the ring. The horizontal and vertical offsets at the end of the line are $\mathcal{O}(1 \mathrm{~m})$. This is small enough, and the line short enough, that no separate tunnel for the abort lines is required.

The choice of vertical bending angle will ultimately be made based on engineering considerations. The maximum possible bending angle using this type of magnet is about $190 \mathrm{mrad}$.

\section{PROPER TREATMENT OF INJECTION/EXTRACTION IN SIMULATION}

In most modelling codes, kicker magnets and septa are treated like corrector magnets, i.e., they change the trajectory of the particles but the coordinate system stays centered around the orbit without the kick. Therefore at the end of the last septum, the coordinate system of the kicked beam is significantly different from the one used in the code, making any calculations rather difficult.

To circumvent this problem, a script was written for AT [1]: It goes through the ring part of the injection or extraction system starting at a place where the injected/extracted beam is still centered. Kickers and septa are replaced by rectangular bending magnets with the proper strength, position and angles. Quadrupoles are replaced by bending magnets with a quadrupole term (again, proper bending strengths and positions are calculated). Even the drift spaces have to be replaced by drift spaces with a slightly different length. To achieve reasonable accuracy the quadrupole magnets need to be sliced, as the transverse position of the beam, and therefore the kick due to the quadrupole field changes significantly over the length of the quadrupole magnet.

This allows construction of a beamline where the coordinate system travels with the injected/extracted beam.

\section{THE MODULAR APPROACH}

When the lines were first designed, the layout was changing several times. This happened because the position of the damping rings with respect to the injector, the RTML and the main linac changed. This required frequent redesigns of the beamlines. To facilitate this, several mod- ules were designed such that the $\beta$-functions at the ends were equal and $\alpha_{x}=\alpha_{y}=\eta_{x}=\eta_{y}=\eta_{x}^{\prime}=\eta_{y}^{\prime}=0$. The modules could then be arranged in an order to roughly correspond to the required layout necessitating only minor rematching. This allowed for a quick change of the layout as at that point only a magnet count and an estimate of the total length of the beamline were required. The different modules are described below:

\section{Ring Connector}

This part serves to cancel the dispersion from the kickers and septa and match the Twiss parameters to the values that all the other modules have at each end. It consists of one bending magnet and six quadrupoles magnets. Twiss parameters are shown in Fig. 2.
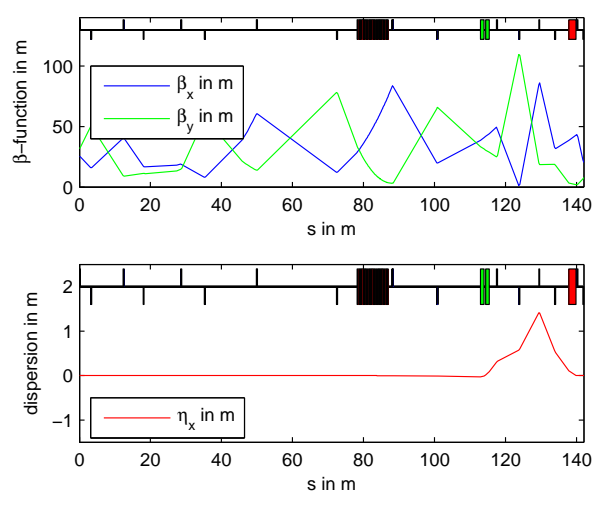

Figure 2: Twiss parameters of the ring connector.

\section{Double Bend Achromat}

The double bend achromat module has the same total bending angle as the ring connector (including kickers and septa). Therefore, it can be used after the ring connector with an opposite bending angle to bend the extracted beam back parallel to the extraction straight. It can also be used to increase the total angle between the ring straight and the extracted beam. If a total angle different from zero or multiples of the achromat bending angle is required, it can easily be rematched to fit the desired bending angle. Twiss parameters are shown in Fig. 3.

\section{FODO Cell}

Usually in a FODO cell the location where $\alpha_{x}=\alpha_{y}=0$ is in the center of the quadrupole magnets. Therefore it is a bit more difficult to build a FODO section with the proper Twiss parameters to be attached to the other modules. This was solved in the following way:

A FODO lattice with half-quadrupoles on each end was designed and matched to the required Twiss parameters. This was the basis for four different cells: 

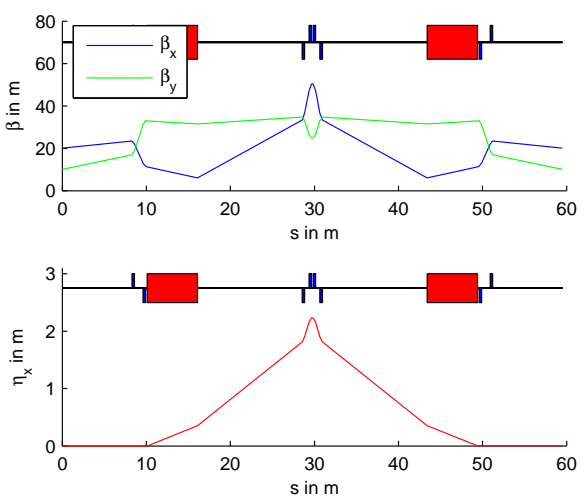

Figure 3: Twiss parameters of the double bend achromat.

1. If only one FODO cell is required, this cell is used. Instead of the half quadrupoles at either end, is has full quadrupoles. It is rematched to still have the nominal Twiss parameters (including $\alpha_{x}=\alpha_{y}=0$ ) at either end.

2. The 'start-FODO' cell: This module also has full quadrupoles at either end. At the entrance it is rematched to have the required Twiss parameters but at the end, the Twiss parameters are still matched to the center of the quadrupole.

3. The 'end-FODO' cell: Here the first half quadrupole is eliminated (but keeping the Twiss parameters as they would be at that place) and the end has a full quadrupole and is rematched to the usual Twiss parameters.

4. The 'middle-FODO' cell: This is the original FODO cell but shifted by half a quadrupole, i.e. no quadrupole at the start but a full quadrupole at the end.

If two FODO cells are required, the 'start-' and 'end-'cell are used. If more than two are required, additional 'middle-'cells can be inserted between the two to get to about the required length of the line. If needed, the whole line can be rematched to achieve a specified length.

\section{CURRENT DESIGN}

\section{Injection and Extraction Line}

With the current design of housing both damping rings in a common tunnel, the length of the injection lines is rather short. All that needs to be done in the lines is to cancel the dispersion from the kickers and to have reasonable Twiss parameters (with $\eta_{x}=\eta_{x}^{\prime}=0$ ). This can be achieved by just using the ring connector described above after the kickers and septa had been moved to appropriate locations in the ring.

\section{Abort Line}

The abort line is currently located downstream from the extraction. When parameters for dedicated kickers and
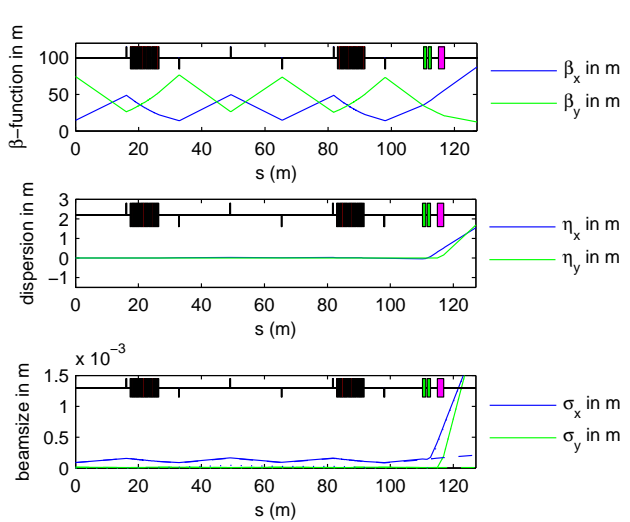

Figure 4: Beam parameters of the abort line for $150 \mathrm{mrad}$ vertical bending angle.

septa become available, the exact position of the kickers and septa will likely change and the lattice in the straight will be reoptimized for them, but this will not change the general features of the abort line. Also, there might be engineering constraints requiring a different location of the dump. Parameters of the current abort line (assuming a vertical bending angle of $150 \mathrm{mrad}$ ) are shown in Fig. 4.

\section{CONCLUSION}

It was important to properly model the kickers and septa as there were quadrupoles interleaved with them. The modular design was very useful to be able to quickly adapt to frequent layout changes without starting from scratch every time. There currently exists a workable solution for all the required lines, although details are bound to change as the design of the ILC complex evolves.

\section{Acknowledgements}

The part of the injection and extraction lines involving the kickers and septa was designed by Aimin Xiao (ANL). The bending magnets used in all lines are based on a bending magnet developed by Peter Tenenbaum (SLAC) for the RTML.

\section{REFERENCES}

[1] A. Terebilo, Accelerator Toolbox for MATLAB [2], SLACPUB-8732 and www-ssrl.slac.stanford.edu/at/.

[2] Matlab [2], The Language of Technical Computing, Programming, Version 7. MATLAB is a registered trademark of The MathWorks, Inc. 